Mit neuer Autorität in Führung 
Frank Baumann-Habersack

\section{Mit neuer Autorität in Führung}

Warum wir heute präsenter, beharrlicher und vernetzter führen müssen

Springer Gabler 
Frank Baumann-Habersack

Hannover-Burgdorf

Deutschland

ISBN 978-3-658-02145-0

ISBN 978-3-658-02146-7 (eBook)

DOI 10.1007/978-3-658-02146-7

Die Deutsche Nationalbibliothek verzeichnet diese Publikation in der Deutschen Nationalbibliografie; detaillierte bibliografische Daten sind im Internet über http://dnb.d-nb.de abrufbar.

\section{Springer Gabler \\ (C) Springer Fachmedien Wiesbaden 2015}

Das Werk einschließlich aller seiner Teile ist urheberrechtlich geschützt. Jede Verwertung, die nicht ausdrücklich vom Urheberrechtsgesetz zugelassen ist, bedarf der vorherigen Zustimmung des Verlags. Das gilt insbesondere für Vervielfältigungen, Bearbeitungen, Übersetzungen, Mikroverfilmungen und die Einspeicherung und Verarbeitung in elektronischen Systemen.

Die Wiedergabe von Gebrauchsnamen, Handelsnamen, Warenbezeichnungen usw. in diesem Werk berechtigt auch ohne besondere Kennzeichnung nicht zu der Annahme, dass solche Namen im Sinne der Warenzeichenund Markenschutz-Gesetzgebung als frei zu betrachten wären und daher von jedermann benutzt werden dürften. Der Verlag, die Autoren und die Herausgeber gehen davon aus, dass die Angaben und Informationen in diesem Werk zum Zeitpunkt der Veröffentlichung vollständig und korrekt sind. Weder der Verlag noch die Autoren oder die Herausgeber übernehmen, ausdrücklich oder implizit, Gewähr für den Inhalt des Werkes, etwaige Fehler oder Äußerungen.

Gedruckt auf säurefreiem und chlorfrei gebleichtem Papier

Springer Fachmedien Wiesbaden ist Teil der Fachverlagsgruppe Springer Science+Business Media (www.springer.com) 


\section{Danksagung}

Zunächst danke ich außerordentlich meiner Lektorin Anne Jacoby und meinem Lektor Erik Prochnow für die Unterstützung bei der Erarbeitung des Buchs. Maria Eilers half mir mit dem „ResonanzSchreiben“ bei der Reflexion während des Schreibprozesses. Und die Grafiken erstellte wie immer professionell und auf den Punkt Meike Vincentz.

Juliane Wagner vom Verlag Springer Gabler wusste ich wohlwollend unterstützend im Hintergrund. Ich bin dankbar für ihre Zuversicht und Ermunterung in das Projekt, insbesondere während einer schwierigen Phase.

Wie wohl jedes Buch, so ist auch dieses durch „geronnenes“ Wissen und ,geronnene Erfahrung“" entstanden.

Meine Lektoren und ich haben darauf geachtet, dass alle Quellen angegeben wurden, die ich verwendet habe. Doch im Laufe meines Lebens bin ich vielen inspirierenden Menschen begegnet, die bei mir etwas bewirkt haben, ohne dass ich dies jetzt konkret einem speziellen Menschen zuordnen könnte.

Somit ein Dank, in alphabetischer Reihenfolge, an diejenigen, von denen ich weiß, dass die Begegnung mit ihnen eine positive Wirkung bei mir auslöste und sie damit zu einer Quelle für mich wurden: Ursula und Helmut Habersack, Franz Arndt-Herold, Karin, Linus und Maya Baumann, Winiger Beuse, Thomas Hartmann, Klaus Schoppmeier, Wolf-Dieter Jakob, Ingrid Kadisch.

Ganz bestimmt sind meine baumann.partner Kolleginnen und Kollegen inspirierend. Ich danke euch für die guten Diskussionen und Gespräche: Christa Beyrer, Nicole du Carrois, Miriam Gross, Carsten Hennig, Malte Hübner, Timm Klotz, Nicole Schober, Andrea Strodtmann.

Ganz besonders herzlich danke ich, erneut in alphabetischer Reihenfolge, meinen Interview-Partnerinnen und Partnern für den doch sehr persönlichen Einblick in ihre Biografie zu Autoritäten und ihre Unterstützung: Dr. Manfred Bodin, Dr. Wolfram von Fritsch, Dr. Veit Görner, Brigitte Brunner-Hoolmans, Martin Kind, Hartmut Ostrowski, Dr. Hannes Rehm, Tina Voß und einer Vielzahl an weiteren Interview-Partnerinnen und Partnern, die gerne ihren Beitrag geleistet haben, jedoch aus Sorge vor möglicher Diskreditierung aufgrund ihrer exponierten Stellung anonym bleiben wollten. Diese Sorge zeigte sich branchenübergreifend. Ein guter Grund mehr für dieses Buch. 


\section{Vorwort}

Der Autoritätsbegriff hat es schwer in unserer Gesellschaft der Gegenwart, vor allem in den letzten Jahrzehnten. Nach dem Missbrauch, der mit Autorität in der ersten Hälfte des letzten Jahrhunderts getrieben worden war und der nicht zuletzt in zwei Kriegen mündete, ist es eigentlich erstaunlich, wie lange er sich noch gehalten hat. Das hat sicher damit zu tun, dass Autorität kein „Konzept“ ist, sondern ein Aspekt von Kultur. Nach Abdankung der verbrecherischen Autoritäten wurde deren Versagen personenbezogen verrechnet. In der Kultur blieb jedoch für lange Zeit noch das Bild bestehen, dass es für ein funktionierendes Gemeinwesen (im Großen wie im Kleinen) „den einen Kopf“ geben müsse, der die Richtung bestimmt und die Schritte dahin vorgibt.

Kulturen wandeln sich bekanntlich langsam. Vor allem lassen sich Kulturen nicht managen, weil sie selbstorganisiert entstehen. Und da ist in Bezug auf Autorität ein interessanter Wandel zu beobachten. In den 1950er bis 1970er Jahren begannen sich die Bilder von Autorität langsam zu wandeln. Nachdem der letzte Bundeskanzler, der noch ein nationalsozialistisches Parteibuch gehabt hatte, abgetreten war, wurden die Stimmen der nächsten Generationen in der Gesellschaft zunehmend lauter vernehmbar. Vielleicht lässt sich die Geburtsstunde eines neuen Verständnisses von Autorität mit der Antrittsrede von Willy Brandt als Bundeskanzler verorten, der davon sprach, er wolle „mehr Demokratie wagen“. Doch der Wandel brauchte Zeit, ein neues Bild von Autorität war noch nicht in Sicht. Das alte hingegen wurde zunehmend demontiert. Die „antiautoritäre Bewegung“ der 1970er Jahre bezog sich kritisch auf den Begriff, wollte ihn abschaffen, doch blieb sie ihm in der Negation verhaftet. Es wurden keine Alternativmodelle angeboten, die dauerhaft tragfähig gewesen wären - im Gegenteil, die freien Kommunen, Kinderläden und so weiter wurden vielfach als bedrohlich erlebt und waren eher dazu angetan, die Rufe nach Rückkehr zum traditionellen Autoritätsbegriff zu stärken, als ihm ein neues, attraktives Bild gegenüberzustellen.

Und doch markieren diese Geschehnisse einen schrittweisen kulturellen Wandel. Er ging mit einer zunehmenden Korrosion des Begriffs „Autorität“ einher, ein Wandel, der sich auf vielen gesellschaftlichen Ebenen zeigte und zeigt, sei es in dem Verständnis von Elternschaft und Erziehung, in der Psychologie oder auch in der Unternehmensführung. 
Die moderne Gesellschaft warf zahlreiche „klassische“ Antworten auf konflikthafte Situationen über Bord, wie die Selbstverständlichkeit männlicher Überlegenheit und Vorherrschaft (das ist alles noch recht nah: Bis 1927 durfte ein Mann seine Frau noch körperlich bestrafen, bis 1954 durfte eine Frau nicht arbeiten, ohne dass der Mann zustimmte), die selbstverständlichen Rechte eines Erstgeborenen, die sich selbst legitimierende Führung „von Gottes Gnaden“ und so weiter.

Blicken wir einmal in die Führungstheorie. Hier werden die „Great man“-Theorien, die Bilder ,,heroischer und charismatischer Führung“, abgelöst von einer Kultur des ,,postheroischen Managements". In dieser Kultur wird Führung nicht mehr durch die Zuweisung formaler Autorität installiert, in der die Untergebenen wie „Marionetten“ die Aufgaben erfüllen, die ihnen zugewiesen werden. Es geht vielmehr darum, Wirtschaft und Unternehmen immer wieder neu zu erfinden. In diesem Prozess der kontinuierlichen Bearbeitung von Unsicherheit stellen sich die Aufgaben von Führung neu. Es geht nicht mehr darum, durch zugewiesene Autorität allein Orientierung zu bieten und heldenhaft die Unsicherheit im Unternehmen zu absorbieren. Anders als der „Macher-Mythos“ nahelegt, ist es nicht die eine Person „oben“, die Ziele definiert und vorgibt. Führung ist nur eine von vielen Kontextbedingungen, die in einem Unternehmen wirksam sind - und manchmal funktioniert ein Unternehmen eher trotz seiner Führung gut als wegen dieser. Kontrolle geht in diesem Bild nicht von einer Person aus, sondern von Zusammenhängen, Verknüpfungen, Beziehungen und Interaktionen, wie Dirk Baecker schreibt ${ }^{1}$. Heute ist Kommunikation ein Schlüsselwort geworden, heute geht es eher darum, Kooperationen anzuregen und zu ermöglichen und die Randbedingungen dafür sicherzustellen. Damit geht ein ganz anderes Verständnis von Führung einher, nicht mehr die einsame Entscheidung an der Spitze ist gefragt, sondern die Fähigkeit, sich klug in Netzwerken bewegen zu können. Qualitäten wie die Fähigkeit zum Gespräch, zur Kontaktaufnahme und zur persönlichen Präsenz kennzeichnen eine Führungspersönlichkeit heute. Es sind Qualitäten, die sich weniger aus der Zuweisung einer Position und Funktion ergeben, als vielmehr aus der Art und Weise, wie die Position ausgefüllt wird.

Die Chancen, die in dieser neuen Kultur liegen, sind unübersehbar. Sie sind ein Zeichen der Reife einer Gesellschaft, in der ein partizipatives Führungsverständnis gilt, das auf der Gleichwertigkeit aller Stimmen aufbaut, in der es im Rahmen des Familienlebens darum geht, Demokratie zu lernen statt mit mehr oder weniger massiver Gewalt zu Gehorsam erzogen zu werden, in der die Gleichwertigkeit der Geschlechter auf allen Ebenen gesellschaftlichen Lebens zu einer Selbstverständlichkeit wird u.v.a.m. Doch gibt es solche konstruktiven Veränderungen nicht zum Nulltarif, wie auch dieses Buch zeigt. Die ständig neu zu definierenden Beziehungen, die Suche nach individuell passenden Lösungen, die neu aufkommenden Abstimmungsbedarfe und der steigende Kommunikationsaufwand bergen immer auch die Gefahr der Überforderung in sich - so wird im vorliegenden Buch eine Studie der OSB Wien zitiert, nach der 50\% der Chefs sich ,völlig leer und kaputt“ fühlen. Da fehlen gute Bilder davon, wie sich die Beziehungen über Organisationsebenen,

${ }^{1}$ Baecker, D.: Organisation als System. Frankfurt a. M.: Suhrkamp 1999, S. 363 
über Generationen hinweg verstehen lassen können. Eine Führungskraft und ihre Mitarbeiter sind keine „Freunde“, genauso wenig wie die Eltern für ihre Kinder „Freunde“ sind (zumindest solange sie nicht erwachsen sind). Aber welches Bild passt dann? Der klassische Autoritätsbegriff hat ausgedient, die Verunsicherung zeigt sich manchmal darin, dass heftig nach seiner Rückkehr gerufen wird - etwa in Sendungen wie der Supernan$n y$, wo Eltern dazu gebracht werden, ihre Autorität mit massiven Interventionen wieder herzustellen.

In dem Kontext dieser neuen Kultur steht der von Haim Omer ins Gespräch gebrachte und von mir mit ihm in Deutschland verbreitete Begriff der „Neuen Autorität“. Er basiert auf völlig anderen Bildern und Prämissen als der traditionelle Begriff. Im Zentrum dieses neuen Verständnisses steht der Begriff der Präsenz: Sei es das Elternhaus, die Schule, die Öffentlichkeit oder das Unternehmen, stets geht es darum, eine Form von „Anwesenheit““ und „Dasein“ zu verwirklichen, die nicht (primär) auf Macht und Durchsetzung gegründet ist, sondern auf Kommunikation, auf Beziehung und Kooperation. Als Führungskraft im Sinne der Neuen Autorität behauptet sich eine Person, wenn sie zum Experten der Gestaltung von Interaktionen wird, wenn sie in der Lage ist, Befugnisse, die mit einer Position verbunden sind, persönlich zu verkörpern, eben „da“ zu sein. Damit fokussiert die Neue Autorität auf etwas grundsätzlich anderes als Kontrolle, Durchsetzung oder Macht, nämlich auf beharrliche Präsenz und Verbundenheit. Die Facetten dieses neuen Verständnisses von Autorität werden in diesem Buch ausgearbeitet. Erstmals wird damit das Konzept, das von uns vor allem für die Unterstützung hilfloser Eltern entwickelt wurde, auf Führung übertragen. Konsequent wird Neue Autorität als „Beziehungsthema“ entwickelt und es wird sorgfältig „durchbuchstabiert“, welche Veränderungen dieses Verständnis im Führungsalltag mit sich bringt.

Ich freue mich sehr, dass auf diese Weise die Versuche von Haim Omer und mir, einen komplexen Wandel unserer Kultur auf vielen Ebenen begrifflich zu fassen, beginnen, ein „Eigenleben“ zu führen und sich weiterzuentwickeln. Ich bin sicher, dass dieses wichtige Buch nicht nur von Führungskräften, sondern auch von Personen, die mit Beratung befasst sind, mit Gewinn gelesen werden wird. Und darüber hinaus ist es für jeden interessant, der sich dafür interessiert, den komplexen Wandel, in dem wir in unserer Gesellschaft stehen, zu verstehen, kritisch nachzuvollziehen und vielleicht weiterzutreiben. In diesem Sinn wünsche ich dem Buch viele engagierte Leserinnen und Leser.

Witten/Osnabrück, im Januar 2015

Prof. Dr. Arist von Schlippe 


\section{Inhaltsverzeichnis}

1 Einleitung: Macht allein führt nicht weiter $\ldots \ldots \ldots \ldots \ldots \ldots \ldots \ldots$

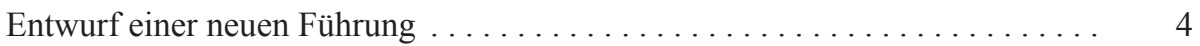

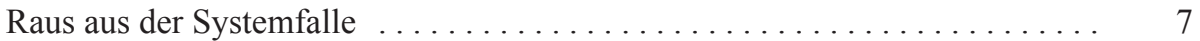

Was Sie in diesem Buch erwartet $\ldots \ldots \ldots \ldots \ldots \ldots \ldots \ldots \ldots \ldots$

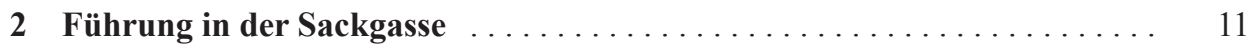

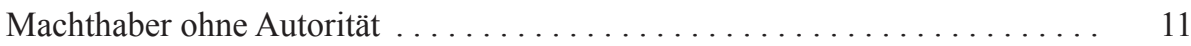

Generation Y pfeift auf Führung alter Schule . . . . . . . . . . . 13

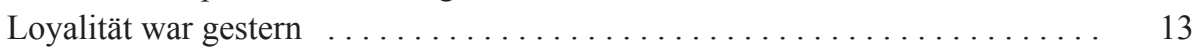

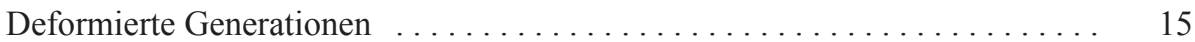

Machtspiele: Narzissmus liebt Intransparenz . . . . . . . . . . . . . 18

Projektchaos: Mangelnde Präsenz begünstigt Burnout . . . . . . . . . . . . 19

Change, Change, Change: Hysterie versus Beharrlichkeit . . . . . . . . 20

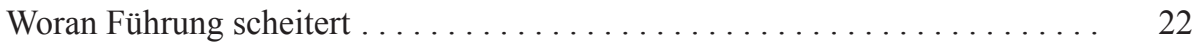

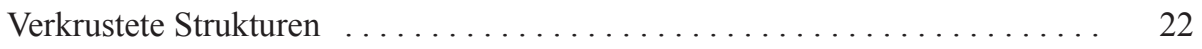

„Mehr vom Gleichen“ führt nicht weiter $\ldots \ldots \ldots \ldots \ldots \ldots \ldots \ldots \ldots \ldots$

Vielfalt schafft Potenziale .......................... 24

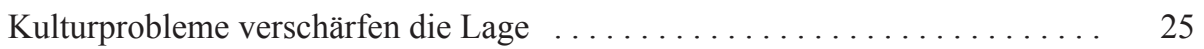

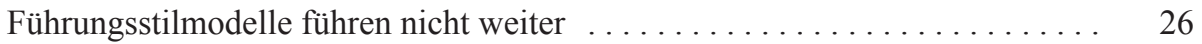

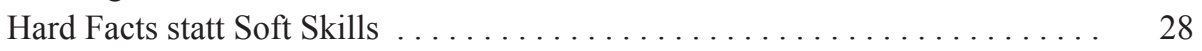

Wundermittel „Bewegliche Strukturen“? . . . . . . . . . . . . . . . 29

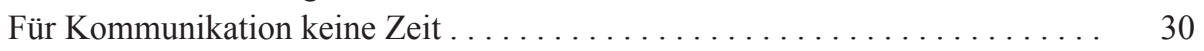

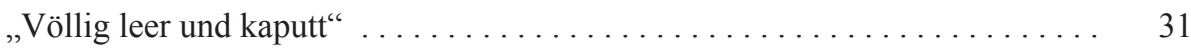

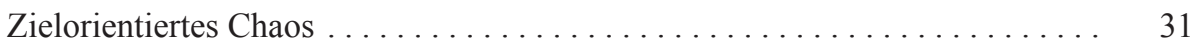

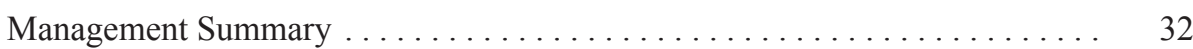

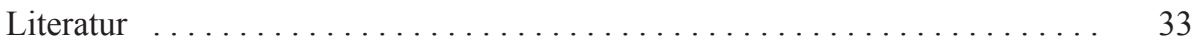




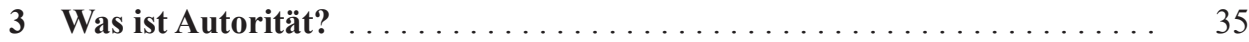

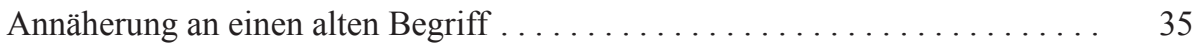

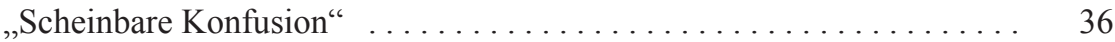

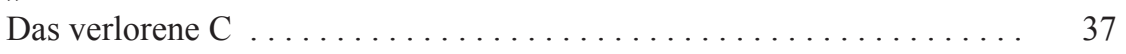

Autorität und Person ......................... 38

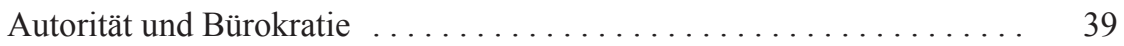

Autorität und Macht . . . . . . . . . . . . . . . . . . 42

Autorität und Gewalt .......................... 43

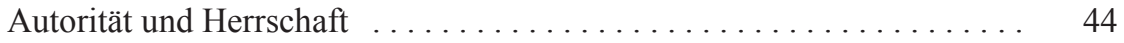

Autorität und Erlösung . . . . . . . . . . . . . . . . . . . . . 45

Zerrbild: Fünf Filter verstellen unseren Blick $\ldots \ldots \ldots \ldots \ldots \ldots \ldots \ldots$

Ich sehe was, das du nicht siehst $\ldots \ldots \ldots \ldots \ldots \ldots \ldots \ldots \ldots \ldots$

Filter: Die eigene Biografie und Rollenmodelle . . . . . . . . . . 50

Filter: Die Persönlichkeitsstruktur . . . . . . . . . . . . . . . . 53

Filter: Das Wertesystem ........................ 58

Filter: Wissen ............................ 61

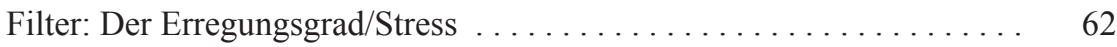

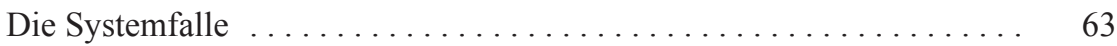

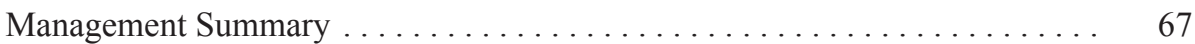

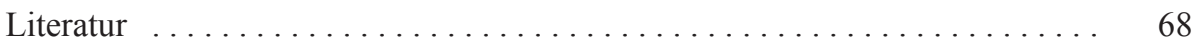

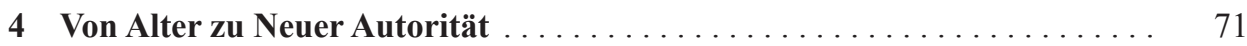

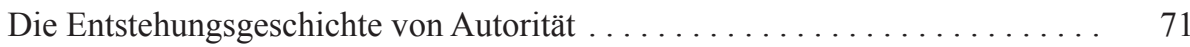

Familie: Vom autoritären Patriarchat zu postheroischen

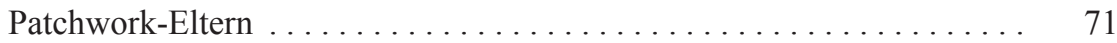

Staat: Von der wilhelminischen Ära zum Merkelismus . . . . . . . . . . . . 76

Schule: Von der Rohrstock- zur Kuschelpädagogik . . . . . . . . . . . . . 78

Wirtschaft: Von den Gründervätern zu agilen Netzwerkern ... . . . . . . 79

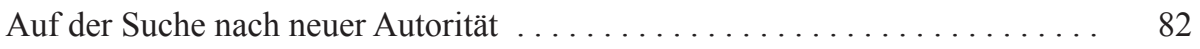

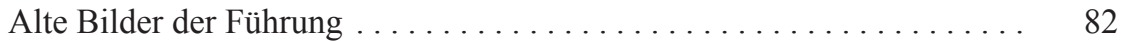

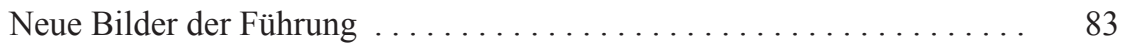

Management Summary . . . . . . . . . . . . . . . . . . . . . . . 85

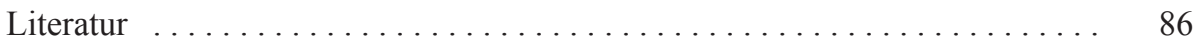

5 Neue Autoritä̈t leben $\ldots \ldots \ldots \ldots \ldots \ldots \ldots \ldots \ldots \ldots \ldots \ldots \ldots \ldots$

Eine Frage der Beziehung . . . . . . . . . . . . . . . . . . . . 89

Zurück zum Miteinander . . . . . . . . . . . . . . . . . . . . . . 90

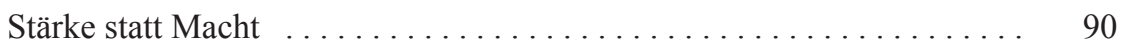

Die Fähigkeit zur Selbstkontrolle $\ldots \ldots \ldots \ldots \ldots \ldots \ldots \ldots \ldots \ldots \ldots$ 
Das Fundament einer neuen Haltung zu Autorität in der Führung:

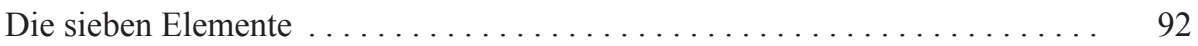

Element 1: Präsenz . . . . . . . . . . . . . . . . . . . . . 94

Element 2: Selbstführung . . . . . . . . . . . . . . . . . . 99

Element 3: Führungskoalition . . . . . . . . . . . . . . . . 102

Element 4: Wiedergutmachung ...................... 104

Element 5: Transparenz . . . . . . . . . . . . . . . . . . . 105

Element 6: Beharrlichkeit und Deeskalation . . . . . . . . . . . . 106

Element 7: Reflexion ........................ 108

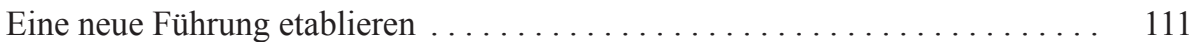

Die individuelle Ebene: Die Macht des Einzelnen zur Veränderung . . . . 111

Entwicklungsfeld 1: Persönlichkeit und Werte . . . . . . . . . . . . 112

Entwicklungsfeld 2: Emotionale Intelligenz $\ldots \ldots \ldots \ldots \ldots \ldots \ldots \ldots$

Entwicklungsfeld 3: Konfliktkompetenz . . . . . . . . . . . . . . . . 119

Entwicklungsfeld 4: Autorität in der persönlichen Biografie . . . . . . 126

Die kulturelle Ebene: Der Unternehmenskompass . . . . . . . . . . . . . 129

Die strukturelle Ebene . . . . . . . . . . . . . . . . . . . . . 132

Paternalistische Kulturen . . . . . . . . . . . . . . . . . . . . 132

Management Summary . . . . . . . . . . . . . . . . . . . . 133

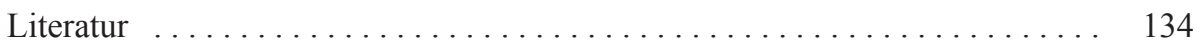

6 Ausblick: In Zukunft führen $\ldots \ldots \ldots \ldots \ldots \ldots \ldots \ldots \ldots \ldots \ldots \ldots$

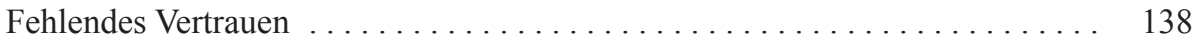

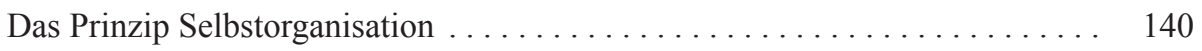

Handlungsfreiheit gewinnen . . . . . . . . . . . . . . . . . . . . 144

Die Chancen sehen .............................. 145

Management Summary . . . . . . . . . . . . . . . . . . . . . . . . 148

Literatur . . . . . . . . . . . . . . . . . . . . . . . . . . 149 


\section{Über den Autor}

Frank Baumann-Habersack ist seit über 10 Jahren geschäftsführender Gesellschafter einer systemischen Organisationsberatung in Burgdorf/Hannover. Neben Führungs-, Konflikt- und Veränderungsthemen gehören Mediation, Coaching und die Gestaltung von Unternehmens- sowie Führungskulturen zu seinen Kernkompetenzen. Der Unternehmer arbeitete zuvor als Führungskraft, Projektleiter und interner Berater. Baumann-Habersack ist Betriebswirt und Arbeitswissenschaftler, Bankkaufmann, Bachelor of Arts, Lehrcoach sowie ausgebildet in NLP, systemischer Familientherapie und Supervision. 